\title{
Prolonged Response to HER2-Directed Therapy in a Patient With HER2-Amplified, Rapidly Progressive Metastatic Colorectal Cancer
}

\author{
Aparna Parikh, MD, MS; Chloe Atreya, MD, PhD; W. Michael Korn, MD; and Alan P. Venook, MD
}

\begin{abstract}
HER2 gene amplifications and activating mutations in the HER2 receptor tyrosine kinase are present in $4 \%$ of metastatic colorectal cancers (mCRCs). HER2-targeted therapy is not standard of care, although preclinical and clinical data suggest that patients with HER2 amplifications and/or HER2-activating mutations may benefit from HER2-directed therapy. HER2 amplifications and activating mutations have also been implicated in resistance to anti-epidermal growth factor receptor-based therapy. This report describes a patient with KRAS, NRAS, and $B R A F$ wild-type $\mathrm{MCRC}$ who experienced disease progression on first-line treatment with FOLFIRI and cetuximab after only 5 months, and subsequently experienced progression on second-line treatment with capecitabine and oxaliplatin plus bevacizumab after 2 months with significant functional decline. Next-generation sequencing of the primary tumor identified HER2 amplification, and we were able to obtain trastuzumab-DM1 for off-label use. The patient had symptomatic clinical benefit from trastuzumab-DM1 and had radiographic disease control for 7 months. On progression, therapy was changed to trastuzumab and pertuzumab, but the patient's disease progressed 3 months later. Treatment with the trastuzumab-DM1 resulted in a sustained response that was longer than his prior responses in the first and second lines of treatment, with a dramatic improvement in the patient's functional status. This case represents the first report, to our knowledge, of successful single-agent treatment of HER2-amplifed CRC with trastuzumab-DM1. Clinical trials targeting patients with HER2mutated and -amplified metastatic colon cancer are currently underway. Molecular insights from investigating HER2 activation and the impact of HER2-directed therapies in a wide variety of solid tumors will create the needed evidence base to more broadly inform patient care.
\end{abstract}

J Natl Compr Canc Netw 2017;15(1):3-8

HER2 gene amplifications and activating mutations in the HER2 receptor tyrosine kinase are present in $4 \%$ of metastatic colorectal cancers (mCRCs). HER2-targeted therapy is not standard of care, although preclinical and clinical data suggest that patients with HER2 amplifications and/or HER2-activating mutations may benefit from HER2-directed therapy. HER2 amplifications and activating mutations have also been implicated in resistance to anti-epidermal growth factor receptor (EGFR)-based therapy.

This report describes a patient with KRAS, NRAS, and BRAF wild-type mCRC who experienced disease

From University of California San Francisco, Hellen Diller Family Comprehensive Cancer Center, San Francisco, California.

Submitted April 29, 2016; accepted for publication September 13, 2016.

Dr. Parikh has disclosed receiving salary from Genentech. Dr. Atreya has disclosed receiving research support from GlaskoSmithKline, Novartis, and Merck, and has disclosed serving in a consulting/advisory role for Genentech, and Bayer Diagnostics. Dr. Korn has disclosed serving in a progression on first-line treatment with FOLFIRI and cetuximab after only 5 months, and subsequently experienced progression on second-line treatment with capecitabine and oxaliplatin plus bevacizumab after 2 months with significant functional decline. Next-generation sequencing (NGS) of the primary tumor identified HER2 amplification and we were able to obtain trastuzumab-DM1 for off-label use. The patient had symptomatic clinical benefit from trastuzumab-DM1 and had radiographic disease control for 7 months. On progression, therapy was changed to trastuzumab and pertuzumab, but the patient's disease progressed 3 months later. Treatment with the trastuzumab-DM1 re- 
sulted in a sustained response that was longer than his prior responses in the first and second lines of treatment, with a dramatic improvement in the patient's functional status.

This case represents the first report, to our knowledge, of successful single-agent treatment of HER2amplifed CRC with trastuzumab-DM1. Clinical trials targeting patients with HER2-mutated and -amplified metastatic colon cancer are currently underway. Molecular insights from investigating HER2 activation and the impact of HER2-directed therapies in a wide variety of solid tumors will create the needed evidence base to more broadly inform patient care.

\section{Case Report}

A 48-year-old Caucasian man presented to his primary care provider with persistent right upper quadrant pain. An ultrasound of the area demonstrated at least 7 heterogeneous lesions throughout the liver. CT and PET imaging confirmed PET-avid liver lesions varying in size from 1 to $6.5 \mathrm{~cm}$. The patient also had many scattered PET-avid pulmonary nodules and a PET-avid rectosigmoid mass with adjacent hypermetabolic lymphadenopathy. A liver biopsy diagnosed metastatic adenocarcinoma (CK20+/CK7+, consistent with a colon primary). Subsequent colonoscopy visualized a nonobstructing, circumferential, ulcerated mass extending 14 to $16 \mathrm{~cm}$ from the anal verge. Biopsy confirmed colorectal adenocarcinoma. The serum carcinoembryonic antigen (CEA) level at the time of diagnosis was $39.5 \mathrm{ng} / \mathrm{mL}$. Molecular analysis demonstrated a KRAS, NRAS, and BRAF wild-type tumor with intact mismatch repair proteins. Further NGS of the primary tumor demonstrated ERBB2 (HER2) and MYC amplification and a TP53 R175H mutation and APC R1386* mutation. Further testing via immunohistochemistry/ fluorescence in situ hybridization (IHC/FISH) for ERBB2 amplification was not performed because testing was not yet standard in CRC and availability of tissue on the liver biopsy was limited.

First-line treatment with FOLFIRI and cetuximab was initiated, and approximately 2.5 months later interval scans showed a modest response in the liver and lungs and stable focal thickening at the rectosigmoid junction (Figure 1). Treatment was continued, but interval imaging in 2 months showed progressive disease in the liver and lungs, and he

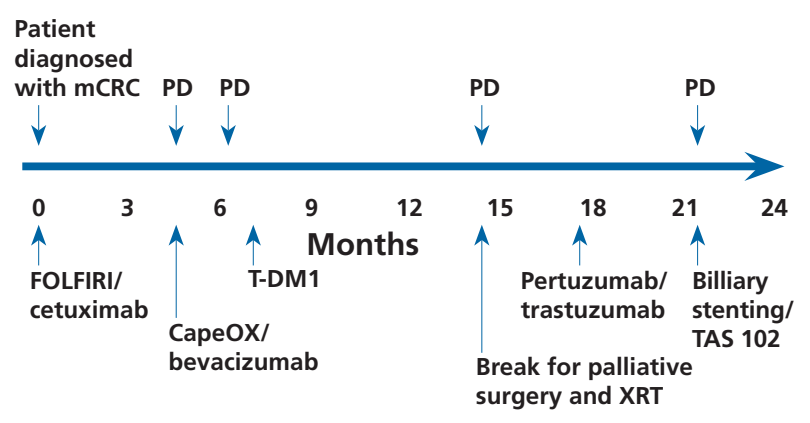

Figure 1 Treatment course.

Abbreviations: CapeOx, capecitabine and oxaliplatin; mCRC, metastatic colorectal cancer; PD, progressive disease; T-DM1, trastuzumab-DM1; XRT, chemoradiation.

was switched to a standard second-line regimen of capecitabine, oxaliplatin, and bevacizumab. Subsequent imaging 2 months later showed continued progression of disease in the liver and lungs, and progressive rectosigmoid luminal narrowing coincident to the patient having worsening right upper quadrant discomfort and more bowel complaints, with increasingly bloody stools and small, frequent bowel movements. His CEA level at the time of progression was $78.9 \mathrm{ng} / \mathrm{mL}$, and he was also complaining of worsening fatigue. Given this rapid progression, off-label treatment with HER2-directed therapy was sought. The patient's insurance provided T-DM1 (trastuzumab-DM1) and he was started on this treatment. Within 4 weeks of starting T-DM1, he showed a marked improvement in functional status and tolerated treatment well except for mild diarrhea. His CEA level at this time was $64.3 \mathrm{ng} / \mathrm{mL}$. Interval imaging at 2 months showed a treatment response in the liver, lungs, and primary tumor. He remained on treatment, and imaging 3 months later showed a continued response in the liver and stable disease in the lungs and the primary tumor (Figure 2). His CEA level reached a nadir at $39.1 \mathrm{ng} / \mathrm{mL}$. Treatment was continued and the patient continued to feel well, with an improvement in energy, and reported less blood in his stools. Interval imaging 2 months later, now 7 months after he began treatment with T-DM1, showed progressive disease in the liver, with an increase in size and number of liver lesions and new biliary ductal dilatation. At this time, the patient was also having more bowel complaints, with decreased stool caliber and severe diarrhea, and treatment was paused in order to address the primary tumor. The patient underwent a palliative laparoscopic diverting sigmoid colostomy and a 10-day 
HER2 Therapy in Metastatic Colorectal Cancer
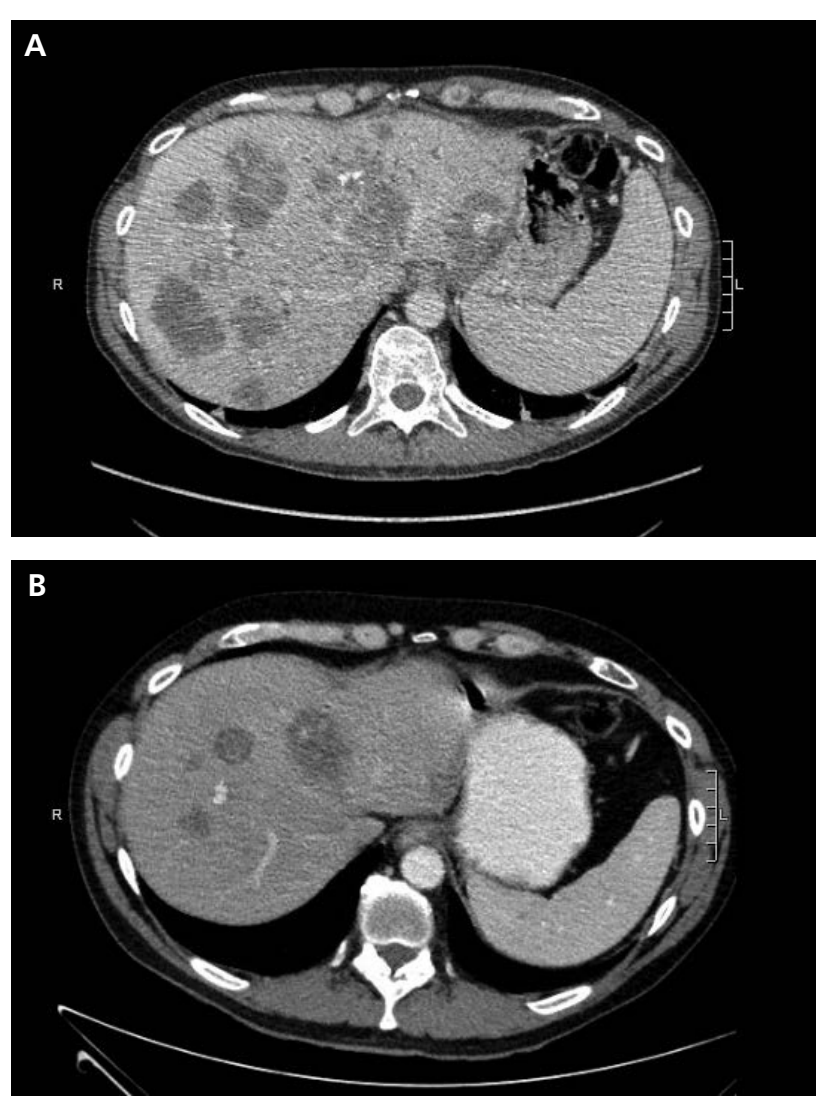

Figure 2 (A) Abdominal CT image before the start of T-DM1 treatment. (B) Abdominal CT image after 5 months of T-DM1 treatment.

course of palliative chemoradiation to the primary because of persistent bleeding. Assessment of circulating cell-free DNA (cfDNA) in the blood at the time of progression showed the TP53 R175H mutation, APC R1386* mutation, and continued detection of HER2 amplification.

After recovery from surgery and radiation, given a longer progression-free interval on third-line therapy with T-DM1 than on first- and second-line treatment and persistent HER2 amplification in cfDNA, authorization was pursued for pertuzumab and trastuzumab. He started treatment with this combination 3 months after T-DM1 was stopped. A new reference laboratory test showed a CEA level of $456 \mathrm{ng} / \mathrm{mL}$ at the time of pertuzumab and trastuzumab initiation. Over the next couple of months, his CEA level remained stable and then increased to $851 \mathrm{ng} / \mathrm{mL}$. Imaging performed after 6 cycles of pertuzumab and trastuzumab showed progressive disease, and HER2directed treatment was stopped. Notably, at the time of progression, another liver biopsy was performed and NGS once again demonstrated a KRAS, NRAS, and BRAF wild-type tumor with MYC amplification and the TP53 mutation but loss of HER2 amplification. After progression, the patient required decompression for biliary obstruction and then started on TAS-102 while pursuing options for further clinical trials.

\section{Discussion}

ERRB2 (HER2/neu) encodes the receptor tyrosine kinase of HER2. ERBB2 gene amplification and oncoprotein overexpression lead to tumor proliferation. HER2 is part of family of 4 related receptor tyrosine kinases-EGFR, HER2, HER3, and HER4involved in activation of several downstream oncogenic pathways, including signaling though PI3K/AKT and MAPK. The HER2/HER3 heterodimer is thought to be one of the most significant contributors to tumor proliferation among the family of receptors. ${ }^{1}$ HER2 testing and HER2-directed therapy have dramatically improved the treatment of HER2positive breast and gastric cancers. ${ }^{2,3}$

Patient selection for HER2-directed treatment is contingent on pathologic determination of HER2 status. HER2 testing in breast and gastric cancers is often a source of confusion, because the staining pattern that defines IHC positivity is different in breast and gastric cancers, and gastric cancers are more heterogeneous in HER2 expression than breast cancers. ${ }^{4}$ In gastric cancer, the largest survival benefit associated with the HER2-targeted monoclonal antibody, trastuzumab, is seen in patients with higher HER2 expression. ${ }^{5}$ Although HER2 amplifications have more recently been recognized as an important driver in several other solid tumors, including mCRC, the definition of HER2 positivity in other solid tumors has not been well-established. ${ }^{4}$ Methodologies for HER2 testing and defining positivity in mCRC are not yet standardized, and efforts to standardize testing in order to identify patients who may be eligible for HER2-directed treatment are underway. ${ }^{6-8}$

It appears that HER2 overexpression is more homogenous in mCRC than in gastric cancer, and is associated with left-sided and rectal primaries. ${ }^{8}$ HER2 overexpression has been noted in $5 \%$ of KRAS wildtype CRC, and data exist suggesting up to a $7 \%$ prevalence of both amplifications and activating mutations. ${ }^{9-11}$ Less is known about HER2-activating 
Parikh et al

mutations in mCRC, but the clinical evidence supporting HER2-directed therapy in $\mathrm{mCRC}$ in both amplifications and activating mutations is evolving and suggestive of efficacy. ${ }^{12-14}$ Notably, approximately $15 \%$ discordance between HER2 expression in colon primaries and metastases has been reported, and HER2 expression may change over time, highlighting the need to further prospectively study the role of HER2 in mCRC. ${ }^{6}$ The prognostic effect of HER2 overexpression in $\mathrm{mCRC}$ remains unclear, with conflicting evidence from retrospective analyses. ${ }^{8,14}$ Another factor yet to be explored in CRC is the interplay of MYC alterations with HER2 overexpression. In early-stage, HER2-positive breast cancer, MYC/chromosome 8 copy number alterations may predict greater benefit from adjuvant treatment with trastuzumab. ${ }^{15}$

In colon cancer, as in this case, HER2 gene amplifications are known to produce resistance to anti-EGFR monoclonal antibodies, such as cetuximab and panitumumab, via activation of the MAPK pathway. ${ }^{16-18}$ There are also several known HER2-activating mutations, such as S310F, L7555, V777L, V842L, and L866M, associated with resistance to anti-EGFR monoclonal antibodies in vitro. Regarding HER2 amplifications, testing in preclinical patient-derived xenograft (PDX) models supports the rationale for dual blockade of HER2/HER3 and EGFR with trastuzum$\mathrm{ab}$ and lapatinib., ${ }^{919}$ Single-agent trastuzumab did not markedly affect HER2 and EGFR phosphorylation but produced dose-dependent suppression of HER3 activation. The addition of lapatinib, a tyrosine kinase inhibitor (TKI) that targets HER2 and EGFR, caused a strong suppression of HER2 but an upregulation of HER3 after a transient blockade, and thus, the 2 together had synergistic inhibitory effects. Singleagent afatinib, an irreversible TKI targeting HER2, EGFR, and HER4, also seems to be as effective as the combination of reversible small molecule TKIs such as lapatinib and trastuzumab. ${ }^{20}$ Experiments using CRC PDX models in HER2-amplified tumors have also explored mechanisms of resistance to TKIs such as lapatinib, and have found that resistance can also be overcome by the addition of pertuzumab, a monoclonal antibody that inhibits HER2 heterodimerization with other HER family members in addition to homodimerization. ${ }^{19}$

In terms of HER2 mutations, HER2-mutant PDX models exhibit tumor regression when treated with dual HER2-targeted therapy consisting of trastu- zumab and a TKI such as lapatinib or neratinib. ${ }^{10}$ Afatinib has also been tested in HER2-mutant colon cancer cell lines. In these cell lines, the tumors were highly sensitive to afatinib but the addition of the HER3 ligand, neuregulin, led to afatinib resistance. In these HER2-mutant CRC cell lines, resistance to afatinib mediated by neuregelin-1 was overcome by pertuzumab, suggesting that pertuzumab may be able to overcome TKI drug resistance mediated by HER3 activation. ${ }^{13}$ Although which pathways and mechanisms of resistance to target remain under exploration in HER2-amplified and -mutated mCRC, additional clinical data supporting the use of HER2directed therapy are emerging.

Trastuzumab was first evaluated in $\mathrm{mCRC}$ in combination with chemotherapy (irinotecan or FLOX) in 2 phase II studies. In these studies there were signals of efficacy, yet low rates of HER2 overexpression in all-comer populations limited further exploration at that time. ${ }^{21,22}$ HERACLES (HER2 Amplification for Colo-Rectal Cancer Enhanced Stratification) was the first large phase II clinical trial to evaluate HER2-directed therapy-lapatinib in combination with trastuzumab-in patients with HER2-amplified mCRC. In HERACLES, HER2 positivity was defined as $3+$ or $2+$ by IHC or FISH-positive (defined by a HER2:CEP17 ratio $>2$ in $>50 \%$ of cells). Lapatinib is approved in combination with chemotherapy in patients with metastatic breast cancer who have progressed on trastuzumab. ${ }^{23}$ The HERACLES study found that patients with HER2-amplified mCRC refractory to standard therapy, including anti-EGFR therapy, had a $35 \%$ response rate to lapatinib plus trastuzumab, with an 8.5-month median duration of response, and a median time to progression of 5.5 months. ${ }^{9}$ In a phase I study of lapatinib and cetuximab in advanced solid tumors, patients with mCRC who responded to this combination included one who had received prior anti-EGFR therapy, suggesting another possible approach to overcoming resistance to anti-EGFR therapy. ${ }^{24}$

Another phase II clinical trial, HERACLESRESCUE, evaluating T-DM1 after progression on trastuzumab and lapatinib, is underway in mCRC. T-DM1 is an antibody drug conjugate whereby trastuzumab is connected via a stable thioether linker to emtansine (DM1), a highly potent microtubule chemotherapy agent. Once trastuzumab binds to HER2expressing cells, the linker is broken down, releasing 
HER2 Therapy in Metastatic Colorectal Cancer

DM1 intracellularly. ${ }^{25,26}$ In patients with metastatic breast cancer whose disease had progressed on trastuzumab and taxane, T-DM1 showed improved overall survival compared with capecitabine and lapatinib. ${ }^{27}$ The rationale for T-DM1 in HERACLES-RESCUE resulted from testing in CRC PDX models among patients with acquired resistance to trastuzumab and lapatinib on the HERACLES study. These PDX models were found to have high levels of HER2 expression, and treatment with T-DM1 resulted in tumor shrinkage, whereas animals treated with pertuzumab alone had no response. In HERACLESRESCUE, acknowledging that HER2 expression may change over time, not only were tumors rebiopsied on progression and tested via IHC/FISH, but also serial liquid biopsies for cfDNA were performed. In the future, as cfDNA is further evaluated for detection of both HER2 amplifications and mutations in CRC, liquid biopsies may help inform HER2-directed therapeutic decision-making over the course of treatment. . $^{27,28}$

Another recently presented study, My Pathway, evaluated trastuzumab plus pertuzumab in HER2amplified and -mutated tumors. Patients were eligible for the study if their tumors were found to have HER2 amplification or an activating mutation via NGS, and/or HER2 amplification via IHC/FISH. The 13 patients with HER2-positive mCRC all had HER2 amplifications and had received a median of 5 lines of previous treatment (range, 2-8 lines), and $54 \%$ had received prior anti-EGFR antibodies. The overall response rate was $38 \%$ and the clinical ben- efit rate was $54 \%$ (defined as complete or partial response or stable disease for at least 6 months if treatment was discontinued, or 5.5 months if treatment was ongoing). The median time to progression was 5.6 months, with ongoing response in $23 \%$ of patients at the time of recent data presentation. ${ }^{11}$

This is the first case, to our knowledge, showing the activity of single-agent T-DM1 in a patient with HER2-amplified mCRC before any other HER2directed treatment. T-DM1 treatment after disease progression on lapatinib and trastuzumab is currently being explored in the HERACLES-RESCUE study and data have not yet been reported.

\section{Conclusions}

It remains to be seen which agent or combinations of agents targeting HER2, HER3, and EGFR, and in what line of therapy, may be most efficacious for patients with HER2-amplified or -mutated mCRC, but targeting this pathway seems to be of increasing clinical importance for these patients. Furthermore, how HER2 positivity should be defined and tested for in $\mathrm{mCRC}$ has yet to be standardized, and this will be an important step toward establishing the evidence base needed to solidify the role of HER2-directed therapy in the treatment paradigm for mCRC. This report illustrates a case of T-DM1 therapy demonstrating remarkable clinical benefit in the third line for a patient with HER2-amplified, refractory mCRC, and supports the ongoing efforts to understand the role of HER2 in mCRC.

\section{References}

1. Hudis CA. Trastuzumab-mechanism of action and use in clinical practice. N Engl J Med 2007;357:39-51

2. Bang YJ, Van Cutsem E, Feyereislova A, et al. Trastuzumab in combination with chemotherapy versus chemotherapy alone for treatment of HER2positive advanced gastric or gastro-oesophageal junction cancer (ToGA): a phase 3, open-label, randomised controlled trial. Lancet 2010;376:687697.

3. Swain SM, Kim SB, Cortes J, et al. Pertuzumab, trastuzumab, and docetaxel for HER2-positive metastatic breast cancer (CLEOPATRA study): overall survival results from a randomised, double-blind, placebo-controlled, phase 3 study. Lancet Oncol 2013;14:461-471.

4. Vakiani E. HER 2 testing in gastric and gastroesophageal adenocarcinomas. Adv Anat Pathol 2015;22:194-201.

5. Bang YJ, Van Cutsem E, Feyereislova A, et al. Trastuzumab in combination with chemotherapy versus chemotherapy alone for treatment of HER2positive advanced gastric or gastro-oesophageal junction cancer (ToGA): a phase 3, open-label, randomised controlled trial. Lancet 2010;376:687697.

6. Lee WS, Park YH, Lee JN, et al. Comparison of HER2 expression between primary colorectal cancer and their corresponding metastases. Cancer Med 2014;3:674-680.

7. Valtorta E, Martino C, Sartore-Bianchi A, et al. Assessment of a HER2 scoring system for colorectal cancer: results from a validation study. Mod Pathol 2015;28:1481-1491

8. Seo AN, Kwak Y, Kim DW, et al. HER2 status in colorectal cancer: its clinical significance and the relationship between HER2 gene amplification and expression. PloS One 2014;9:e98528.

9. Siena A, Sartore-Bianchi A, Lonardi S, et al. Trastuzumab and lapatinib in HER2-amplified metastatic colorectal cancer patients (mCRC): the HERACLES trial [abstract]. J Clin Oncol 2015;33(Suppl):Abstract 3508.

10. Kavuri SM, Jain N, Galimi F, et al. HER2 activating mutations are targets for colorectal cancer treatment. Cancer Discov 2015;5:832-841.

11. Hurwitz H, Hainsworth JD, Swanton C, et al. Targeted therapy for gastrointestinaI (GI) tumors based on molecular profiles: early results from MyPathway, an open-label phase Ila basket study in patients with advanced solid tumors [abstract]. J Clin Oncol 2016;34(Suppl 4S):Abstract 653.

12. Marx AH, Burandt EC, Choschzick M, et al. Heterogenous high-level HER-2 amplification in a small subset of colorectal cancers. Human Pathol 2010;41:1577-1585.

13. Bose R, Ali S, Jain N, et al. Frequency of HER2 mutations and amplification in GI malignancies and ability of pertuzumab to overcome neuregulin1 mediated drug resistance to a HER2 tyrosine kinase inhibitor in colon cancer [abstract]. J Clin Oncol 2016;34(Suppl 4S):Abstract 630. 
Parikh et al

14. Park DI, Kang MS, Oh SJ, et al. HER-2/neu overexpression is an independent prognostic factor in colorectal cancer. Int J Colorectal Dis 2007;22:491-497.

15. Perez EA, Jenkins RB, Dueck AC, et al. C-MYC alterations and association with patient outcome in early-stage HER2-positive breast cancer from the north central cancer treatment group N9831 adjuvant trastuzumab trial. J ClinOncol 2011;29:651-659.

16. Vlacich G, Coffey RJ. Resistance to EGFR-targeted therapy: a family affair. Cancer Cell 2011;20:423-425.

17. Bertotti A, Papp E, Jones S, et al. The genomic landscape of response to EGFR blockade in colorectal cancer. Nature 2015;526:263-267.

18. Yonesaka K, Zejnullahu K, Okamoto I, Satoh T. Activation of ERBB2 signaling causes resistance to the EGFR-y. Sci Transl Med 2011;3:99ra86.

19. Bertotti A, Migliardi G, Galimi F, et al. A molecularly annotated platform of patient-derived xenografts ("xenopatients") identifies HER2 as an effective therapeutic target in cetuximab-resistant colorectal cancer. Cancer Discov 2011;1:508-523.

20. Leto SM, Sassi F, Catalano I, et al. Sustained inhibition of HER3 and EGFR is necessary to induce regression of HER2-amplified gastrointestinal carcinomas. Clin Cancer Res 2015;21:5519-5531.

21. Ramanathan RK, Hwang JJ, Zamboni WC, et al. Low overexpression of HER-2/neu in advanced colorectal cancer limits the usefulness of trastuzumab (Herceptin) and irinotecan as therapy. A phase II trial. Cancer Invest 2004;22:858-865.
22. Clark JW ND, Hollis D, Mayer R. Phase-II trial of 5-fluororuacil (5-FU), leucovorin $(\mathrm{LV})$, oxaliplatin $(\mathrm{Ox})$, and trastuzumab $(\mathrm{T})$ for patients with metastatic colorectal cancer (CRC) refractory to initial therapy. Onkologie 2003;26(Suppl 3):13-46.

23. FDA Approval for Lapatinib Ditosylate. National Cancer Institute Web site. Available at: http://www.cancer.gov/about-cancer/treatment/drugs/fdalapatinib. Accessed April 21, 2016.

24. Deeken JF, Wang H, Subramaniam D, et al. A phase 1 study of cetuximab and lapatinib in patients with advanced solid tumor malignancies. Cancer 2015;121:1645-1653.

25. Barok M, Joensuu $H$, Isola J. Trastuzumab emtansine: mechanisms of action and drug resistance. Breast Cancer Res 2014;16:209.

26. Junttila TT, Li G, Parsons K, et al. Trastuzumab-DM1 (T-DM1) retains all the mechanisms of action of trastuzumab and efficiently inhibits growth of lapatinib insensitive breast cancer. Breast Cancer Res Treat 2011;128:347356.

27. Verma S, Miles D, Gianni L, et al. Trastuzumab emtansine for HER2-positive advanced breast cancer. N Engl J Med 2012;367:1783-1791.

28. Siena S, Bardelli A, Sartore-Bianchi A, et al. HER2 amplification as a 'molecular bait' for trastuzumab-emtansine (T-DM1) precision chemotherapy to overcome anti-HER2 resistance in HER2 positive metastatic colorectal cancer: The HERACLES-RESCUE trial [abstract]. ] Clin Oncol 2016;34(Suppl 4S):Abstract TPS774. 